\title{
Vasodilators: minoxidil and drugs used in peripheral vascular and cerebral disorders
}

\author{
GORDON D O LOWE
}

Vasodilator drugs relax vascular smooth muscle, either directly or indirectly through adrenergic neurones. Different vasodilators may act principally on arteries, principally on veins, or on both arteries and veins. Dilatation of arterioles reduces peripheral vascular resistance and hence lowers arterial blood pressure. Dilatation of veins increases peripheral venous pooling of blood, reducing venous return to the heart and tending to lower cardiac output and arterial blood pressure. Reflex sympathetic tachycardia tends to restore cardiac output and blood pressure.

There is sound rationale and proof of efficacy for using vasodilators in three disorders-hypertension, angina, and heart failure. In hypertension blood pressure is lowered by reducing peripheral vascular resistance. In angina the reductions in venous return (reducing preload) and arterial blood pressure (reducing afterload) reduce the workload of the left ventricular myocardium and hence reduce its requirement for oxygen. In heart failure reduction of the workload of the ventricles improves their performance. Of the newer vasodilators, prazosin, calcium antagonists, and captopril are covered in separate articles in this series. Minoxidil, reviewed here, is a recently introduced arterial vasodilator for treating severe hypertension. Although the efficacy of vasodilators in peripheral and cerebral vascular disease has not been proved, new drugs continue to be marketed and these are considered later in this article.

\section{Minoxidil (Loniten)}

Minoxidil is an arterial vasodilator that acts directly on smooth muscle in the vessel wall. Lowering of blood pressure results in a reflex increase in sympathetic activity, causing tachycardia and increased cardiac output. As with hydralazine, a beta-adrenergic blocking drug is routinely given with minoxidil to control tachycardia and to aid reduction in blood pressure. A dose of 80-160 $\mathrm{mg}$ propranolol or $100 \mathrm{mg}$ atenolol daily is usually sufficient. Methyldopa or clonidine may be substituted if beta-adrenergic blockers are contraindicated. Because of the increase in sympathetic activity, minoxidil is contraindicated in phaeochromocytoma and should be used with caution in angina.

Minoxidil also causes pronounced fluid retention, and hence a diuretic must also be given. A thiazide diuretic may be sufficient if renal function is normal, but a more powerful "loop" diuretic such as frusemide is required if renal function is impaired or if oedema or weight gain appear when taking a thiazide diuretic. Oedema should be controlled before starting minoxidil and weight should be measured at each visit.

University Department of Medicine, Royal Infirmary, Glasgow G4 OSF

GORDON D O LOWE, MB, MRCP, lecturer and honorary senior registrar
Oral administration of minoxidil results in near complete absorption, with a peak plasma concentration at about 60 minutes and a maximal effect on blood pressure within a few hours. The plasma half life is four hours, but the effect on blood pressure is prolonged for up to three days owing to tissue binding of the drug. Most of the drug is cleared by liver metabolism, hence little or no adjustment is required in renal failure. In patients receiving peritoneal dialysis or haemodialysis, however, minoxidil is best given after dialysis since it is easily dialysible.

Adverse effects of minoxidil include weight gain, oedema, tachycardia, and worsening of angina or heart failure as noted above. Hypertrichosis (increased hair, especially on the face and arms) occurs in all patients receiving long term treatment and may be unacceptable, especially to women. On the other hand, some men have reported regrowth of hair on bald patches. The mechanism of hypertrichosis is not established, but no hormonal changes have been reported. All patients should be warned about its inevitable occurrence before starting treatment. After withdrawal of the drug the abnormal hair usually disappears within several weeks. Some women, however, prefer to continue minoxidil in preference to other second line antihypertensive drugs, and control hypertrichosis by shaving or using depilatory creams, applied every one to six weeks.

Many patients develop changes in the $T$ wave on electrocardiography. These are usually asymptomatic and disappear on continued treatment. Pericardial effusion, again often asymptomatic, has been described, particularly in patients with uraemia, who are prone to this disorder in the absence of minoxidil: a cause and effect relationship has been suggested. Fatal pericardial effusion has been reported. Less common side effects include rashes, gastrointestinal upset, and breast tenderness.

Because of its side effects and the need for concomitant use of a beta-adrenergic blocker and a diuretic, minoxidil has been reserved for the treatment of severe or moderately severe hypertension in patients who do not respond to, or who are intolerant of, conventional treatment with up to three first line antihypertensive agents-for example, diuretics, beta-adrenergic blockers, hydralazine, clonidine, or methyldopa. Minoxidil is given once or twice daily, starting with $5 \mathrm{mg}$ a day and increasing by $5-10 \mathrm{mg}$ every three to seven days. The usual maximum dose is $50 \mathrm{mg}$ a day, but in exceptional cases doses of up to $100 \mathrm{mg}$ daily have been used. More rapid increase in dosage may be given in hospital to patients with severe hypertension under close observation - for instance, $5 \mathrm{mg}$ every four to six hours.

Wide experience with minoxidil has confirmed that it is usually effective in controlling severe and resistant hypertension. With careful monitoring, adverse effects are usually acceptable, and many patients comment favourably on the lack of drowsiness, impairment of sexual function, or postural or exercise induced hypotensive dizziness, which are such troublesome side effects of other potent hypotensive agents such as adrenergic neurone blockers. The number of tablets taken daily is also often fewer with a regimen of minoxidil, beta-blocker, and diuretic than 
with other combinations of antihypertensives. In the occasional patient who is resistant to minoxidil, or, more commonly, in the patient who does not tolerate side effects, captopril or prazosin may be substituted. Rebound hypertension after withdrawal of minoxidil has not been observed: in fact the hypotensive effect may continue for several weeks.

\section{Vasodilators in peripheral vascular and cerebral disorders}

Vasodilators, old and new, are still prescribed in great quantity (and at great expense) for treating Raynaud's phenomenon, chronic occlusive arterial disease of the leg, and "cerebral vascular disease" - which is often a misnomer for any organic mental impairment in the elderly. There is little sound rationale or proof of efficacy for use of vasodilators in these disorders, especially the latter two. Table I lists vasodilators that have been used in treating peripheral vascular or cerebral disorders, or both.

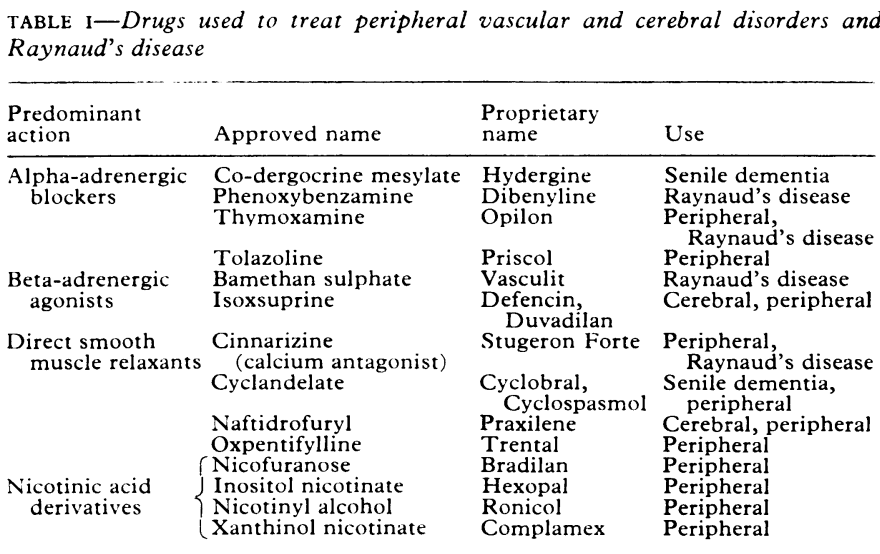

Vasodilators may act by a direct effect on vascular smooth muscle-for instance, by inhibiting intracellular phosphodiesterase to increase cyclic adenosine- $5^{\prime}$-monophosphate or block calcium entry-or indirectly through the sympathetic control of blood vessel tone (table I). In normal subjects alpha-adrenergic blockers increase skin blood flow, while beta-adrenergic agonists increase muscle blood flow. Some drugs may act at more than one site.

In subjects with local ischaemia of the limb or brain the therapeutic aim of vasodilator treatment is selective increase of blood flow in the ischaemic area. There is little evidence that vasodilators have such an effect. This is not surprising when you consider that in chronic occlusive arterial disease of the leg or brain ischaemia arises from fixed, atherosclerotic stenosis rather than vasospasm. Similarly, structural arterial changes are present in many people with severe Raynaud's phenomenon-for example, that associated with scleroderma. Since vasodilators produce generalised vasodilatation, theory predicts that they should in fact decrease blood flow in ischaemic regions by "stealing" blood into other regions that are inappropriately vasodilated. In practice this has often been shown. For example, the potent vasodilator, epoprostenol (prostacyclin, $\mathrm{PGI}_{2}$ ) decreases cerebral blood flow. Vasodilators can cause systemic hypotension, which increases the resistance of collateral vessels and decreases blood flow. Conversely, increase in systemic blood pressure lowers collateral resistance and increases collateral blood flow.

In addition to vasodilatation, there is evidence that some of the newer drugs may reduce blood viscosity and increase red cell filterability (especially oxpentifylline), stimulate the fibrinolytic capacity of plasma, or decrease platelet aggregation. Furthermore, naftidrofuryl affects intracellular metabolism, producing increased concentrations of adenosine triphosphate
(ATP) and decreased concentrations of lactic acid under ischaemic conditions. Whether any of these additional effects on the blood or on metabolism are advantageous in peripheral vascular and cerebral disorders is not yet established.

Table II gives predictable side effects of vasodilators and cautions in their use. Flushing, headache, and gastrointestinal disturbance are observed more often with some vasodilators than others, possibly because of selective actions on blood vessels in the skin, head, and gut. Nicotinic acid derivatives may produce hyperglycaemia and should therefore be used with caution in diabetes. Sinus bradycardia with worsening of cerebrovascular symptoms has been reported with co-dergocrine mesylate.

TABLE II-Adverse effects of vasodilators and cautions in their use

Adverse effects
Hypotensive dizziness and faints, especially postural and exercise ińduced
Tachycardia, palpitations
Flushing, headache
Nausea, vomiting
Cautions
Angina, recent myocardial infarction
Bradycardia, heart block
Recent haemorrhage
Other hypotensive drugs

Other hypotensive drugs

\section{RAYNAUD'S PHENOMENON}

The mainstay of treatment in Raynaud's phenomenon is avoidance of cold, which precipitates the ischaemic attacks. Uncontrolled studies have reported that some adrenergic neurone blockers and alpha-adrenergic blockers may increase digital blood flow and improve symptoms in some people, but their use is limited by side effects (table II). Recent reports suggest similar responses to intermittent infusions of the potent prostaglandin vasodilators, prostaglandin $\mathrm{E}_{1}$ and epoprostenol; treatment with the orally administered anabolic steroid, stanozolol, which increases fibrinolysis; and the rather time consuming and expensive procedure of plasma exchange. The drug effects could be placebo responses in a disorder of variable severity influenced by emotional factors. One hundred and twenty years after Raynaud described the disorder, rational evaluation of drug treatment by randomised double blind trials is only just beginning. Recent preliminary reports of double blind, placebo controlled studies of calcium antagonists and prostacyclin infusions are encouraging. Application of vasodilator drugs to the fingers in ointments may reduce systemic side effects. A recent report of benefit from topical glyceryl trinitrate in a placebo controlled trial suggests that further evaluation of topical vasodilators is worth while.

Raynaud's phenomenon has been reported in association with several drugs-oral contraceptives, beta-adrenergic blockers, antimigraine preparations derived from ergot, methysergide, bromocriptine, bleomycin, and captopril.

\section{INTERMITTENT CLAUDICATION}

The appearance of intermittent claudication indicates a high risk of death from coronary or cerebral arterial disease in the next few years. In contrast, the outlook for the limbs is relatively good: about $70 \%$ of patients improve or remain stable, and fewer than $10 \%$ come to major amputation. Symptoms improve with cessation of smoking and with regular exercise, and patients should be repeatedly encouraged in these two actions of proved value. Reconstructive surgery is indicated in a few patients who do not improve appreciably with conservative treatment. Drug treatment is difficult to evaluate: placebo controlled double blind trials have shown improvement in up to half of the placebo group. Regular observation of their own symptoms and regular exercise may partly explain this placebo response. While a few 
controlled trials have suggested greater improvement on active drug than on placebo, any differences seem marginal and of doubtful benefit to patients. Blood pressure and flow in the leg after exercise are as likely to decrease as increase on vasodilator treatment.

\section{ISCHAEMIC REST PAIN AND PREGANGRENE}

Even in severe ischaemia a considerable percentage of patients improve on conservative treatment. In one trial two thirds of patients claimed a short term improvement in rest pain after placebo injections of saline. Evaluation of treatment is therefore difficult. In severe ischaemia vessels are already maximally dilated as a result of local release of vasoactive metabolites from ischaemic tissue. Vasodilators are unlikely to improve flow and may worsen ischaemia by "stealing" blood flow to other tissues. Controlled trials do not support their use. Recent trials of naftidrofuryl are more encouraging, and an increase in tissue $\mathrm{pH}$ in the skin of the ischaemic foot suggests that its metabolic effects on ischaemic tissue may be beneficial. Nevertheless, its place in management remains to be established by further trials using endpoints directly beneficial to the patient. Similar trials are required for the prostaglandin vasodilators, prostaglandin $\mathrm{E}_{1}$ and epoprostenol. These potent vasodilators also inhibit platelet aggregation, and there is evidence that antiplatelet drugs may benefit some patients with ischaemic toes and fingers who have evidence of increased platelet activity.

\section{CEREBRAL DISORDERS}

It has been said that a man is as old as his arteries, but current opinion is that most old people with dementia have idiopathic neuronal degeneration (senile dementia or Alzheimer's disease) rather than cerebrovascular disease. Cerebral blood flow is decreased in dementias, but this is more likely to reflect the decreased metabolic requirements of the atrophied brain than ischaemia. Dementia associated with atherosclerosis often has a "stepwise" clinical pattern, associated with multiple cerebral infarcts. It seems unlikely that such "all or none" events could be modified by vasodilators. "Stealing" of blood to other tissues on vasodilator treatment is a big risk, as the brain is likely to suffer from postural hypotension: syncope and stroke may result. Once again, assessment of drugs is difficult. Many demented patients respond to the increase in attention resulting from clinical trials. Meaningful endpoints are difficult to define and measure. Few double blind trials have studied large numbers of patients for more than 12 weeks. As in claudication, any differences between active drug and placebo appear marginal and of doubtful benefit to patients. Several practical textbooks of geriatric medicine do not even mention vasodilators. It is much more important to detect treatable causes of confusion in the elderly (drugs, heart block, heart failure, infections, thyroid disorder, vitamin $B_{12}$ deficiency) and to ensure adequate support $\mathscr{g}$ for the demented and their relatives.

I thank Dr Christopher Isles for helpful discussions on minoxidil.

\section{Bibliography}

Pettinger $W$ ed. The Brook Lodge conference on minoxidil. 7 Cardiovasc Pharmacol 1980;2, suppl 2:S91-S246.

Symposium proceedings, including pharmacology, clinical experience (largely American), effects on renal function, and side effects.

Mackay A, Isles C, Henderson I, Fife R, Kennedy AC. Minoxidil in the management of intractable hypertension. $Q \mathcal{F} \mathrm{Med}$ (new series) 1981; 50:175-90.

Experience with 87 patients in Glasgow, including useful review of side effects.

Blunt RJ, Porter JM. Raynaud syndrome. Semin Arthritis Rheum 1981;10: 282-308.

Reviews aetiology, diagnosis, and management, with full list of references (260).

Boobis LH, Bell PRF. Can drugs help patients with lower limb ischaemia? Br F Surg 1982;60 suppl:S17-S23.

Readable, comprehensive, and up to date review of chronic ischaemia of the leg. Includes clinical course, aetiology, and all categories of drug treatment. Good source of references.

Meehan SE, Walker WF. Naftidrofuryl for severe ischaemia: assessment using skin $\mathrm{pH}$ micro-electrode measurements. Curr Med Res Opin 1982;7:690-9.

Improvement in skin tissue $\mathrm{pH}$ in patients without established skin changes in a controlled study. Reviews metabolic effects of the drug that may be responsible.

Hyams DE. Cerebral function and drug therapy: the use of cerebral vasodilators, "vasorelaxants," "haemokinators," and "activators." In: Brocklehurst JD, ed. Textbook of geriatric medicine and gerontology. Edinburgh: Churchill Livingstone, 1978:670-711.

Comprehensive review of cerebral circulation, metabolism, and related drugs. Tabulates results of double blind controlled trials. Full list of references (about 500).
A previously healthy man of 75 has developed a fine tremor of both hands. There is no pill-rolling tendency, and the tremor is not present during sleep. Walking is normal, and the tremor disappears during purposeful movements. All reflexes are normal and there are no sensory changes. Might these symptoms be the result of Parkinson's disease or of hereditary benign essential tremor?

I suspect that this is a form of senile tremor and, as such, a variant of so-called essential tremor. Such tremors are usually most noticeable when the hands are held outstretched and often are not so evident during purposeful movements. They are usually more of an embarrassment to the patient than anything else, though they may interfere with writing and in drinking from a full glass. Essential tremor is not accompanied by any other neurological signs. The absence of any other signs of change in tone, akinesia, or loss of associated movement would be against a parkinsonian tremor, which is usually a coarse rather than a fine tremor. As all tremors disappear during sleep this phenomenon is of no diagnostic value. - N E F CARTLIDGE, consultant neurologist, Newcastle upon Tyne.

A young woman was recently diagnosed as suffering from thyrotoxicosis and diabetes. Is there any link between these two diseases?

Yes. About a third of patients with thyrotoxicosis have glycosuria and an abnormal oral glucose tolerance test result, usually of the lag type. The latter suggests that the mechanism is increased absorption of carbohydrates, but rapid metabolic turnover of sugars probably also plays a part. The abnormal tolerance to glucose usually disappears with treatment of the thyrotoxicosis, but if blood sugar concentrations exceed $12 \mathrm{mmol} / \mathrm{l}$ after glucose or two hours after a meal then diabetes is likely. A family history of diabetes is also more frequent when the two diseases coexist.-ALEX PATON, postgraduate dean, NE Thames region.

$A$ vegetarian, who takes cheese, butter, and dried milk but not fresh milk, claims that fermented soya beans contain sufficient vitamin $B_{12}$ for his needs. Is this so?

The requirement for vitamin $B_{12}$ is low, the recommended dietary allowance being set in the United States as $3 \mu \mathrm{g} /$ day. ${ }^{1}$ Nutritional deficiency from an inadequate dietary intake is rare and is most likely to occur in those who maintain a strict vegetarian diet. Microbial synthesis of the vitamin is believed to be the origin of the vitamin $B_{12}$ in animal tissues, and there is extensive synthesis by the flora in the human large bowel. Probably, therefore, a person consuming a lactovegetarian diet and fermented products may be getting sufficient vitamin $B_{12}$ from his diet.-D A $T$ southgate, head, Division of Nutrition and Food Quality, Norwich.

\footnotetext{
National Research Council. Recommended dietary allowances. 9th ed. Washington: National Academy of Sciences, 1980.
} 